\title{
Using MODIS Normalized Difference Vegetation Index to monitor seasonal and inter-annual dynamics of wetland vegetation in the Great Artesian Basin: a baseline for assessment of future changes in a unique ecosystem
}

\author{
Caroline Petus ${ }^{*}$, Megan Lewis and Davina White \\ School of Earth and Environmental Sciences, The University of Adelaide, Adelaide 5005, Australia - \\ caroline.petus@gmail.com, (megan.lewis,davina.white)@adelaide.edu.au
}

KEY WORDS: Environment, Vegetation, Mapping, Monitoring, Analysis, Satellite, Change

\begin{abstract}
:
The Great Artesian Basin mound springs (Australia) are unique wetland ecosystems of great significance. However, these unique ecosystems are endangered by anthropogenic water extraction. Relationships have been established between the vegetated wetland area and the discharge associated with individual springs, providing a potential means of monitoring groundwater flow using measurements of wetland area. Previous studies using this relationship to monitor Great Artesian Basin springs have used aerial photography or high resolution satellite images, giving sporadic temporal information. These "snapshot" studies need to be placed within a longer and more regular context to better assess changes in response to aquifer draw-downs. In this study, the potential of medium resolution MODIS Normalized Difference Vegetation Index data for studying the long-term and high frequency temporal dynamics of wetland vegetation at the Dalhousie Spring Complex of the GAB is tested. Photosynthetic activity within Dalhousie wetlands could be differentiated from surrounding land responses. The study showed good correlation between wetland vegetated area and groundwater flow, but also the important influence of natural species phenologies, rainfall, and human activity on the observed seasonal and inter-annual vegetation dynamic. Declining trends in the extent of wetland areas were observed over the 20002009 period followed by a return of wetland vegetation since 2010. This study underlined the need to continue long-term medium resolution satellite studies of the Great Artesian Basin as these data provide a good understanding of variability within the wetlands, give temporal context for less frequent studies and a strong baseline for assessment of future changes.
\end{abstract}

\section{INTRODUCTION}

The Great Artesian Basin (GAB) of Australia is one of the largest groundwater resources in the world (Ponder, 2002), underlying about one fifth of the Australian continent (Figure 1a). Groundwater from the GAB supports a series of mound spring which contain a number of rare and relic endemic flora and fauna (Fensham and Fairfax 2003; Gotch et al., 2008; Ponder, 2002). The GAB springs are of great national and international importance for their ecological, scientific and economic values, and are culturally significant to indigenous Australians. In the recent decade, the sustainability of these groundwater-dependent ecosystems has become uncertain and numerous cases of spring extinction have been observed. In the near future, demands for this precious groundwater resource are likely to be considerable, particularly from proposed mining and petroleum activities, (Fensham et al., 2010; Mudd, 1998). It is thus particularly important to develop reliable methods for monitoring the long term artesian flow to inform management of the GAB and better protect this sensitive environment and its ecosystems.

Direct measurements of spring flow are notoriously difficult and expensive if precise estimates are required (Niejalke and Lamb, 2001). Relationships have been established between the wetland area associated with individual springs and their discharge (L.s ${ }^{1}$ ), providing a potential means of monitoring groundwater flow using measurements of vegetated wetland area (Fatchen, 2001; Fatchen and Fatchen, 1993; White and Lewis, 2011; Williams and Holmes, 1978). Previous studies using this relationship to monitor Great Artesian Basin springs have used sporadic aerial photography or high resolution satellite images calibrated to Normalized Difference Vegetation Index (NDVI) (Niejalke and Lamb, 2001, White and Lewis, 2011). These intermittent high resolution studies have only given "snapshots" of vegetated wetland area dynamics at selected springs in the DSC. They need to be placed within a longer and more regular context to better assess changes in response to aquifer draw-downs (White and Lewis, 2011).

Since the launch of the MODIS satellite in 1998, medium resolution $(250-m)$ MODIS NDVI time-series data have been applied successfully to quantify vegetation activity and to monitor vegetation dynamics (e.g. Jacquin et al., 2010; Jönsson et al., 2010) at high temporal frequency (16 days composites). In this study, 8 to 10 years of MODIS NDVI images were used to monitor and quantify vegetation activity and dynamics of the Dalhousie Spring Complex wetlands located on the southwest margin of the Great Artesian Basin (Figure 1a and b). DSC is the most important spring complex in the GAB and discharges $90 \%$ of total spring flow in South Australia through 148 separate spring vents (Gotch, 2010). Recent field work (March 2009) increased knowledge about wetland vegetation associated with active Dalhousie springs (White and Lewis, 2010a, b). The dominant vegetation comprises reeds and sedges or tall reeds dominated by Phragmites australis (Common Reed) and openforest, woodland and open woodland dominated by Melaleuca glomerata (White Tea-tree) trees 10-12 m tall. Ephemeral vegetation in spring tails mainly comprises mixed sedgelands including Cyperus spp. and Juncus sp.

* Corresponding author 
The aim of this study was to test the potential of MODIS NDVI data as long-term tracer of wetland vegetation in the DSC. Particular objectives were to identify and map the spring vegetation greenness, to produce and analyse time traces of NDVI and wetness indices, as well as to relate information about temporal variability of springs derived from medium resolution remote sensing to groundwater flow, rainfall, ecological processes and human management

\section{METHODS}

MODIS-Aqua NDVI images centred on DSC were acquired between July 2002 and May 2010, providing continuous 16-day composite data over approximately 8 years. MODIS data were resampled to WGS 84 / UTM zone 53 and maps of the study area were produced (Figure 1c). With reference to air photos, field observation and site-specific knowledge (White and Lewis, 2010a, b), 21 MODIS pixels representative of six different typical vegetation and land cover types at DSC were geographically located on the MODIS-Aqua images. Four of these pixels were representative of Melaleuca glomerata, three of Phragmites australis, and two of ephemeral vegetation. For comparison, four pixels representatives of saline, three of upland and five of riverine cover types were also selected. These ecosystems are the typical non-wetland ecosystems encountered around the DSC (figure 1a).

Time-traces of NDVI were extracted from these pixels and analysed with a Non-Metric Multidimensional Scaling (NMDS) ordination to determine whether the wetland types could be differentiated on the basis of the NDVI sequence, and if so, to identify the NDVI factors contributing to the separation. The $90^{\text {th }}$ percentile (P90) of the NDVI datasets were calculated in order to determine a NDVI threshold value discriminating photosynthetic activity of the characteristic wetland vegetation species in the Dalhousie Springs from surrounding land responses. Mean Monthly NDVI time traces were also computed from the distinctive Melaleuca glomerata, Phragmites australis and ephemeral vegetation NDVI values recorded between 2002 and 2010.

In order to study the extent (in $\mathrm{km}^{2}$ ) of the vegetated wetland area in the Dalhousie Springs Complex, the number of pixels with NDVI values over the selected threshold was computed and converted to area $\left(\right.$ Area $\left._{\mathrm{NDVI}}, \mathrm{km}^{2}\right)$ of vegetated wetland for each of the 16-day composite MODIS-Aqua images. Complementary MODIS-Terra NDVI images between February 2000 and July 2002 were also processed to extend the period of wetland area recording.

The intra and inter-annual patterns of the Area ${ }_{\mathrm{NDVI}}$ index were then related to the seasonal wetland vegetation changes, as well as to rainfall $(\mathrm{mm})$ and groundwater flow discharge $\left(\mathrm{L} . \mathrm{s}^{-1}\right)$ data furnished respectively by the Australian Government Bureau of Meteorology and the South Australian Department of Environment and Natural Resources. As flow gauge data are sparse and not available for the whole Dalhousie Springs, comparisons between the vegetated wetland area and the groundflow data were conducted at a large spring (DAA01) within DSC where flows were recorded between 2002 and 2010 (Figure 1c). Furthermore, as flow measurements are not available regularly at this spring (1 or 2 measures/year), comparison of mean annual discharge data and Area $_{\mathrm{NDVI}}$ were performed. (Figure 1c, DAA001). Finally, rainfall measurements were selected from the Hamilton Station, the closest meteorologic station to Dalhousie ( $80 \mathrm{~km}$ west).
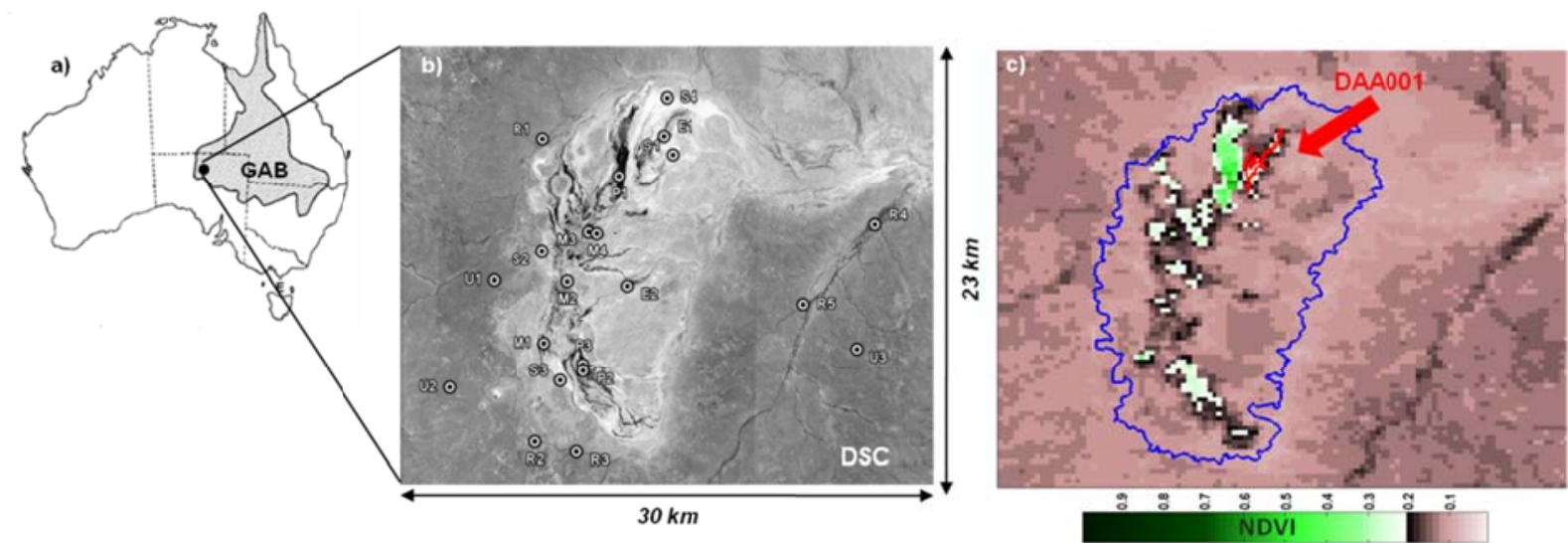

Figure 1: a) Extent of the Great Artesian Basin in Australia and location of the Dalhousie Spring Complex. b) Location of selected pixels: Dalhousie Spring vegetation (P: Phragmites; M: Melaleuca; E: Ephemeral) and surrounding lands (S: Saline; U: Upland; R: Riverine). c) Example of MODIS NDVI map. Spring discharge boundaries for the whole DSC and the spring DAA001 are indicated by blue and red lines, respectively. Boundaries are from White and Lewis (2011). 


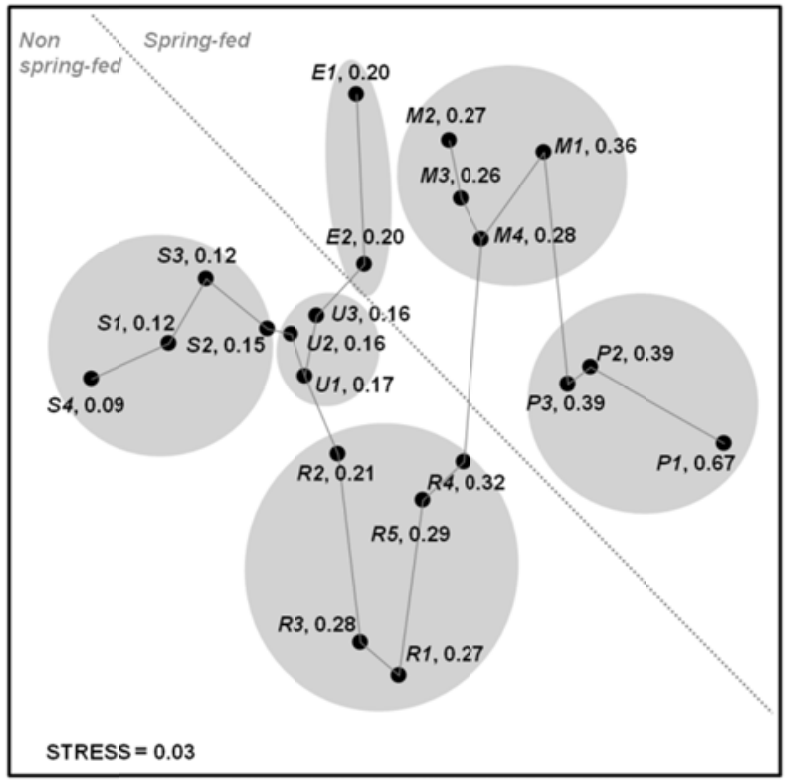

Figure 2: Non-Metric Multidimensional Scaling (NMDS) ordination plots of the 21 ground points based on Bray-Curtis distance calculated with the NDVI values from 2002 to 2010. Pixel names as referenced on Figure 1 and $90^{\text {th }}$ percentile (P90) values calculated from the NDVI time series of the different cover type pixels are indicated in black: Pixel_Name, P90_value. The stress value close to 0 underlines the high quality of this NMDS representation.

\section{RESULTS}

\subsection{Delineating wetland extent}

Figure 2 presents the NMDS plot based on Bray-Curtis distance calculated with the 8-year NDVI values (2002 to 2010) extracted from the 21 respective pixels. The principal feature evident is the good grouping of sample sites representative of same wetland or land type (Figure 2).

The vegetation communities indicative of the DSC are well differentiated from the surrounding non-spring fed land covers in the ordination. In terms of NDVI values, the wetland vegetation are characterized by P90 values systematically equal or greater than 0.2 , with maximal NDVI values registered for the Phragmites australis sites (Figure 2). Surrounding non-spring-fed dryland responses exhibit lower NDVI values $(0.09<\mathrm{P} 90<0.17)$, excepted at the riverine sites $(\mathrm{P} 90>0.21)$ (Figure 2$)$

On the basis of these observations a threshold of 0.2 was established, above which NDVI values are considered to be indicative of wetland vegetation. As the riverine ecosystem pixels tested exhibit P90 values over 0.2 (Figure 2), a mask was applied to the MODIS images to confine analysis to the Dalhousie Spring discharge area of our study zone (Figure 1c) and exclude surrounding riverine ecosystems.

\subsection{Temporal dynamics of wetland extent}

Figure 3 presents the temporal analysis of wetland vegetation areas (combined ephemeral, Melaleuca and Phragmites). Comparative analyses (not shown here) have been conducted to confirm compatibility between the AreaNDVI calculated from the Aqua and Terra NDVI products. All analyses were conducted on the whole DSC discharge area, except comparison with groundflow data, which was conducted for the individual spring DAA01 (Figure 1c). Times series analysed are of 8 or 10 years.

Multi-annual values of AreaNDVI implemented using the 0.2 NDVI threshold are highly dynamic and characterized by an overall decreasing trend over the 2000-10 period, but with a slight return of wetland vegetation since 2010 (Figure 3). This index ranges from 5.77 to $107 \mathrm{~km}^{2}$, with notable maxima recorded before 2002 by the MODIS-Terra satellite (Figure 3a).

A relationship between groundwater spring flow and wetland area is observed for the spring DAA001 (Figure 1c and 3b). Multi-annual (2002-2010) vegetation areas and flow values are clearly correlated, except during years 2007 to 2009. Excluding these exceptional years, $79 \%$ of the AreaNDVI variance is explained by the multi-annual mean flow variability at DAA001 (Figure 3b). The lack of relationship observed between flow data and wetland vegetation from 2007 to 2009 can be explained by an extensive fire started by a lightning strike in 2007 at spring DAA001. This fire led to a marked decrease in the vegetation extent with a slow return of vegetation only in 2010 (T. Gotch, pers. comm.).

At the multi-annual scale, changes in vegetation extent are also strongly correlated to magnitude of preceding rainfall (Figure 3c). Six-monthly smoothed rainfall and AreaNDVI values recorded between 2000 and 2010 show similar trends. Extended wetland areas recorded before 2002 respond to high rainfall totals whereas the minimum vegetation extent observed in 2008 is clearly linked to dry climatic conditions recorded this year (370 mm cumulative rain in 2000 vs. 129 $\mathrm{mm}$ in 2008). 

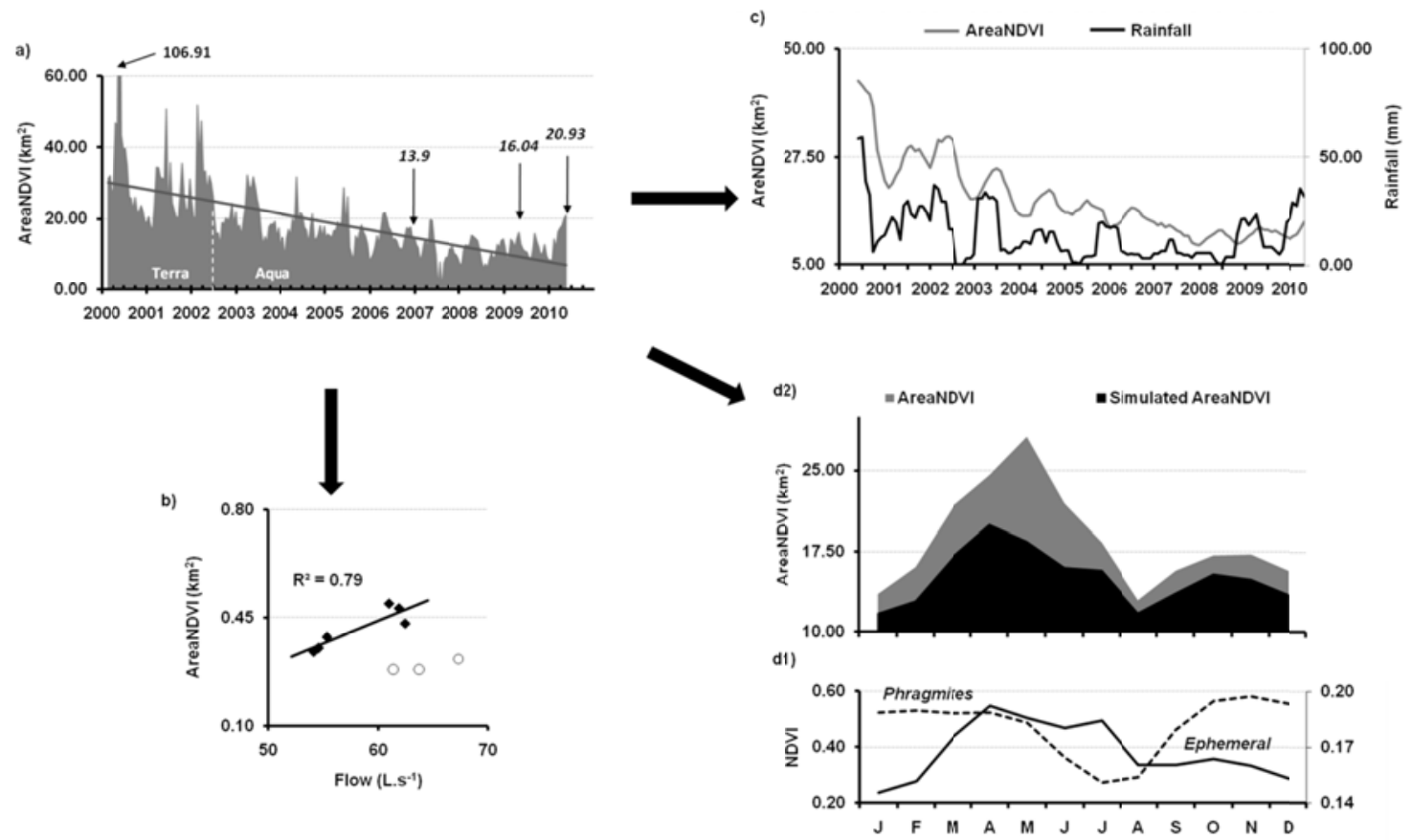

Figure 3: Study of wetland area vegetation extends implemented using the 0.2 NDVI threshold. a) Multiannual values of AreaNDVI recorded between February 2000 and May 2010 (MODIS-Terra and -Aqua data) and linear trendline. b) Correlation between Area $_{\mathrm{NDVI}}$ and spring flow recorded between 2002 and 2010 for the spring DAA001 (Figure 1c). White circles indicate years 2007, 2008, and 2009. c) Comparison between 6-months smoothed rainfall and Area NDVI values recorded between 2000 and 2010. d1) Mean monthly NDVI values calculated from NDVI times traces (2002 to 2010) recorded at 2 selected Phragmites australis and ephemeral pixels (P1 and E2, Figure 1b) d2) Comparison between the simulated mean monthly Area ${ }_{\text {NDVI }}$ reconstructed from the P1 and E2 monthly signal (c1) and the measured mean monthly Area ${ }_{\mathrm{NDVI}}$.

\section{DISCUSSION}

Satellite imagery provides the synoptic view necessary for inventory and mapping of ecological systems (Hewitt and Mason, 1990). The different spatial and temporal resolutions of sensors are complementary for conducting comprehensive environmental monitoring. This study demonstrates the potential of 250-m MODIS Normalized Difference Vegetation Index for studying long-term dynamics of Dalhousie Springs wetland vegetation.

\subsection{Delineating wetland extent}

The scale, heterogeneity and complex spatial patterning of the dispersed wetland vegetation posed challenges for this MODIS NDVI study. Selection of pixels representative of homogeneous vegetation stands was difficult and required expert knowledge of the environment. However, even if the number of samples was limited by the landscape (Figure 1b), their distinctiveness is supported by the Non-Metric Multidimensional Scaling (Figure 2), as the six DSC cover types fall into six distinctive clusters. Intra-group variability can be attributed to sample site differences resulting from variation between vegetation stands, intermixing of species and variable influences of soil background: the scale and heterogeneity of the wetlands made it difficult to find homogeneous patches that would fill the MODIS instantaneous ground resolution.

Despite these limitations, our study demonstrates that springfed vegetation photosynthetic activity can be differentiated from surrounding land responses in this medium resolution imagery. The higher P90 values recorded in photosynthetic activity traces are good indicators of wetland vegetation, when riverine ecosystems are excluded (Figure 2). Similar results were obtained by White et al. (2011), using NDVI data derived from a QuickBird image and field vegetation percentage cover. These authors also found low NDVI signal for the GAB surrounding dryland vegetation, medium signal for the ephemeral wetland ecosystems and higher NDVI values for the perennial wetland samples composed by Phragmites australis, and Melaleuca glomerata. The distinct differences in NDVI of the different vegetation types evident were attributed to the contrasting canopy structure and growth patterns between the different communities (White et al., 2011). This difference between non spring-fed and wetland vegetation greeneess signal allows the establishment of a NDVI threshold value of 0.2 , above which NDVI values are considered to be indicative of wetland vegetation.

\subsection{Temporal dynamics of wetland extent}

Our study confirms the potential of photosynthetic wetland vegetation as an indicator of volume of the spring flow (Fatchen, 2001; Fatchen and Fatchen, 1993; Mudd, 1998; White and Lewis, 2011; Williams and Holmes, 1978), as correlations are demonstrated between multi-annual mean extent of vegetated spring areas and groundwater discharges for one large spring at DSC (Figure 3b). The temporal frequency and archive of MODIS NDVI measurements allow us to demonstrate this relationship with more observations over a longer period than previous studies. 
However, the dynamics of green wetland areas are also controlled by rainfall events on the GAB, natural intra-annual variability of species present within the wetlands, and by historical events at particular sites. Thus, multi-annual variations in vegetation extent are clearly related to the total rain measured at the Hamilton Station (Figure 3c). Ephemeral wetland and Phragmites phenologies explain the mean monthly variations observed in the area index calculated (Figure 3d). Anthropogenic actions, like uncontrolled fire, also play a crucial part in temporal dynamics of spring wetland vegetation (Figure 3a).

Therefore, short-term variations observed in vegetated wetland extent must be interpreted with care and placed in a longer context if they are to be used as indicators of spring flow modification. Long-term medium resolution satellite studies provide a good understanding of variability and a strong baseline for assessment of changes, and give temporal context for less frequent high resolution studies. For example, White and Lewis (2011) record a substantial increase in wetland area at DCS using three epochs of high resolution satellite imagery between 2006 and 2010). The MODIS index of wetland area (AreaNDVI) also shows an increase of about 50\% (Figure 3, AreaNDVI = 13.90, 16.04 and $20.93 \mathrm{~km}^{2}$ ) over these three dates (December 2006, May 2009 and May 2010), but in addition provides a fuller temporal context for these records: 2006 follows six years of declining wetland area, there was further decline in 2007 and 2008, with some increase in wetland area in 2010.

While the MODIS NDVI spatial resolution is not sufficient to give precise measurements of wetland area; it does give objective, repeatable measurements of relative changes in area, and provides new understanding of intra and interannual variability. Variations in the DSC wetland extent observed over the ten years are the result of the combined phenological, natural and anthropogenic influences at different springs throughout the complex. Nevertheless, the trends documented in Figure 10b are quite alarming as they suggest decreases of a third in the area index since 2002 and a return of wetland vegetation since only 2010.

MODIS photosynthetic activity monitoring in association with higher resolution temporally targeted studies must be extended to give a fuller picture of the long term dynamics and trends for DSC. Temporal analyses such as those implemented in this paper help define the range of natural variation in the spring-fed wetlands, and could form the basis for thresholds of acceptable change for environmental management purposes. Change in wetland area beyond such thresholds could provide timely warning of responses to changing aquifer pressures or climate change. Further research at smaller groups of GAB springs is testing the limits imposed by the MODIS NDVI spatial resolution, and will extend our understanding of the spring wetland dynamics.

\section{CONCLUSIONS}

The aim of this study was to test the potential of MODIS NDVI data as long-term tracer of wetland vegetation in the Dalhousie Spring Complex of the Great Artesian Basin. Results obtained proved that photosynthetic activity within DSC wetlands can be discriminated from surrounding land responses in this medium resolution imagery. Up to ten years of wetland vegetation extent were documented. The study reaffirmed the correlation existing between vegetated areas and groundwater flow, but also demonstrated the important influence of rainfall, natural species phenologies, and human activity on the observed seasonal and inter-annual vegetation dynamics. Over the whole Dalhousie Spring discharge area, declining trends in the extent of wetland areas were observed over the 2000-2009 period followed by a return of wetland vegetation since 2010 . This study underlined the need to continue long-term medium resolution satellite studies of the GAB to fully understand variability and trends in the springfed wetlands. Complemented by high resolution studies, use of MODIS imagery over several decades could become a powerful tool for monitoring potential impacts of aquifer drawdown and climate changes in the Great Artesian Basin.

\section{REFERENCES}

Fatchen, T., 2001. Vegetated wetland area as an index of mound spring flows, 4th Mound Spring Researchers Forum, National Parks and Wildlife Service, Adelaide, South Australia, pp. 5-8.

Fatchen, T.H., Fatchen, D.H., 1993. Dynamics of vegetation on mound springs in the Hermit Hill region, Northern South Australia. Western Minning Corporation (Olympic Dam Operations) Pty. Ltd., Adelaide.

Fensham, R.J., Fairfax, R.J., 2003. Spring wetlands of the Great Artesian Basin, Queensland, Australia. Wetlands Ecology and Management 11, 343-362.

Fensham, R.J., Ponder, W.F., Fairfax, R.J., 2010. Recovery plan for the community of native species dependent on natural discharge of groundwater from the Great Artesian Basin., in: East, C. (Ed.), Basin Report to Department of the Environment, Water, Heritage and the Arts, Canberra. Queensland Department of Environment and Resource Management, Brisbane, pp. 1-58.

Gotch, T.B., 2010. Great Artesian Basin Springs of South Australia: Spatial distribution and elevation map. Last updated June 2010 South Australian Arid Lands Natural Resources Management Board.

Gotch, T.B., Adams, M., Murphy, N.P., Austin, A.D., 2008. A molecular systematic overview of wolf spiders associated with Great Artesian Basin Springs in South Australia: evolutionary origins and an assessment of metapopulation structure in two species. Invertebrate Systematics, 22, 151165 .

Hewitt, J.H., Mason, J., 1990. Synoptic inventory of riparian ecosystems: The utility of Landsat Thematic Mapper data. Forest Ecology and Management 33-34, 605-620.

Holland, S.M., 2008. Non-metric multidimensional scaling (MDS) Department of Geology, University of Georgia, Athens.

Jacquin, A., Sheeren, D., Lacombe, J.-P., 2010. Vegetation cover degradation assessment in Madagascar savanna based on trend analysis of MODIS NDVI time series. International Journal of Applied Earth Observation and Geoinformation 12, S3-S10.

Jönsson, A.M., Eklundh, L., Hellström, M., Bärring, L., Jönsson, P., 2010. Annual changes in MODIS vegetation indices of Swedish coniferous forests in relation to snow dynamics and tree phenology. Remote Sensing of Environment 114, 2719-2730.

Mudd, G.M., 1998. Mound springs of the Great Artesian Basin in South Australia: a case study from Olympic Dam. Environmental Geology 39, 463 - 476. 
Niejalke, D., Lamb, K., 2001. Application of a remote sensing tool to assess spring wetland area-preliminary results, 4th Mound Spring Researchers Forum, National Parks and Wildlife Service, Adelaide, South Australia, pp. 16-19.

Ponder, W.F., 2002. Ponder, W.F. (2002) Desert springs of the Australian Great Artesian Basin. In: Sada, D.W. and Sharpe, S.E. (eds.), Conference Proceedings, Spring-fed Wetlands: Important Scientific and Cultural Resources of the Intermountain Region, 7-9 May 2002, Las Vegas, pp. 1-13.

White, D., Lewis, M.M., 2010a. Mapping the spectral and spatial characteristics of mound spring wetland vegetation in South Australia: a novel spectrally segmented PCA approach, Proceedings American Society for Photogrammetry and Remote Sensing Annual Conference, San Diego, California, USA.

White, D., Lewis, M.M., 2010b. Mapping the spectral and spatial characteristics of mound spring wetland vegetation: a novel integrated hyperspectral approach, Proceedings 15th Australian Remote Sensing and Photogrammetry Conference, Alice Springs, Australia.

White, D., \& Lewis, M.M. (2011). A new approach to monitoring spatial distribution and dynamics of wetlands and associated flows of Australian Great Artesian Basin springs using QuickBird satellite imagery. Journal of Hydrology, 408, 140-152

Williams, A.F., \& Holmes, J.W. (1978). A novel method of estimating the discharge of water from the Mound Springs of the Great Artesian Basin, central Australia. Journal of Hydrology, 38, 263-272

\section{ACKNOWLEDGEMENTS}

This research was conducted as part of the project Allocating Water and Maintaining Springs in the Great Artesian Basin funded by the Australian Government National Water Commission and South Australian Government. The MODIS NDVI data were downloaded from the Warehouse Inventory Search Tool (WIST) NASA web site. The assistance of project members is gratefully acknowledged: Brad Wolaver in providing spring flow data, and Travis Gotch for detailed knowledge of spring ecological and management histories. We also thank the Witjira National Park Co-management Board and the traditional owners and native title holders of the Dalhousie Springs country for the opportunity of studying this highly important environment. 